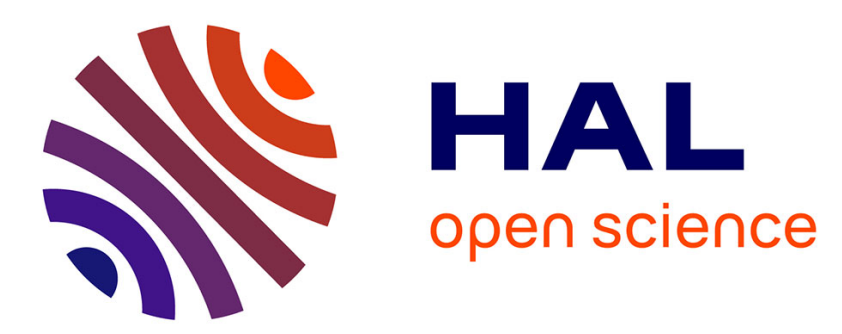

\title{
Simple and double Hopf bifurcations in aeroelastic oscillators with Tuned Mass Dampers
}

Vincenzo Gattulli, Fabio Di Fabio, Angelo Luongo

\section{To cite this version:}

Vincenzo Gattulli, Fabio Di Fabio, Angelo Luongo. Simple and double Hopf bifurcations in aeroelastic oscillators with Tuned Mass Dampers. Journal of The Franklin Institute, 2001, 338 (2-3), pp.187-201. hal-00798950

\section{HAL Id: hal-00798950 \\ https://hal.science/hal-00798950}

Submitted on 11 Mar 2013

HAL is a multi-disciplinary open access archive for the deposit and dissemination of scientific research documents, whether they are published or not. The documents may come from teaching and research institutions in France or abroad, or from public or private research centers.
L'archive ouverte pluridisciplinaire HAL, est destinée au dépôt et à la diffusion de documents scientifiques de niveau recherche, publiés ou non, émanant des établissements d'enseignement et de recherche français ou étrangers, des laboratoires publics ou privés. 


\title{
Simple and double Hopf bifurcations in aeroelastic oscillators with tuned mass dampers
}

\author{
Vincenzo Gattulli, Franco Di Fabio, Angelo Luongo \\ Dipartimento di Ingegneria delle Strutture, delle Acque e del Terreno, Universitá di l'Aquila, \\ 67040 Monteluco Roio, Italy
}

\begin{abstract}
The effects of an added mass on the oscillations of a SDOF bluff body, elastically supported, exposed to a steady flow and undergoing galloping oscillations, are investigated. The stability boundaries of the trivial equilibrium position of the 2DOF system are determined in a four parameters space. The occurrence of different types of bifurcation on these boundaries is highlighted, namely, simple Hopf, non resonant double Hopf and 1:1 resonant double Hopf. The perturbation multiple scale method is employed to analyze the system posteritical behavior around the codimension 1 and codimension 2 critical manifolds. The analytical results are compared with numerical solutions obtained through direct integration of the equations of motion. Finally, the effects of the closeness of the critical frequencies on the non resonant double Hopf manifold, are discussed by using a quasi resonant asymptotic solutions.
\end{abstract}

Keywords: Structural control; Wind engineering; Nonlinear oscillations; Bifurcations; Post critical behavior; Perturbation methods

\section{Introduction}

Flow-induced vibrations constitute important design criteria for many structures subjected to wind and/or ocean waves. It is well established that in the process of 
vibrations induced by flow, the nonlinear interaction effects can be as important as the effects of steady flow loads. Some of these observed nonlinear phenomena are the results of the dynamical interaction between the flow and the structures and should be distinguished from the response of structures to the random effects of the flow turbulence. The commonest interaction phenomenon is the vortex shedding, caused by the organized wake vortex systems forming around the body; the other important one, is the galloping, where a bluff body experiences a classical dynamical instability, leading to oscillations of increasing amplitude in a direction normal to the flow (see e.g. $[1,2])$. Interaction between the two forms of oscillations, is also possible, under certain conditions, e.g. Corless and Parkinson [3].

Recently, many passive and active devices have been proposed aiming to reduce flow-induced oscillations [4-7]. Among them, the tuned mass damper (TMD) has received particular attention. The concept of damping a structural system by adding a small mass to it dates back to the beginning of the century. Many authors have proposed different methods to design the spring-viscous connection of the added mass to the system for reaching a suitable tuning to the frequency of an assigned mode, see e.g. [8,9]. The effects of the others modes of the system have recently been investigated either in forced oscillations, [10], than in self-excited oscillations [11].

Previous studies are mainly devoted to prevent galloping by increasing the critical value at which the phenomenon is triggered. However, in order to investigate the system performance when the wind velocity exceeds this value, an analysis of the post-critical behavior is needed. The present paper deals with analyses of the TMD effects on the galloping of a SDOF aeroelastic oscillator. The multiple scale method is used to investigate the post-critical scenario, where both simple and double Hopf bifurcations are likely to occur. The results are useful to evaluate the effectiveness of TMDs even in the postcritical range.

\section{Equations of motion}

The equations governing the cross-wind oscillations of an elastically supported bluff body connected with a small added mass and subject to a steady flow are derived (Fig. 1). Both the bluff body primary system (PS) and the added mass (TMD) are assumed to posses a SDOF and to be linear; moreover the aerodynamic forces acting on the TMD are assumed to be negligible in comparison with those acting on the PS. Therefore, the only source of nonlinearities arise from these latter. The equations of motion are

$$
\begin{aligned}
& m_{s} \ddot{q}_{1}+2 m_{s} \omega_{s} \xi_{s} \dot{q}_{1}+2 m_{t} \omega_{t} \xi_{t}\left(\dot{q}_{1}-\dot{q}_{2}\right)+m_{s} \omega_{s}^{2} q_{1}+m_{t} \omega_{t}^{2}\left(q_{1}-q_{2}\right)=f_{\mathrm{a}}, \\
& m_{t} \ddot{q}_{2}+2 m_{t} \omega_{t} \xi_{t}\left(\dot{q}_{2}-\dot{q}_{1}\right)+m_{t} \omega_{t}^{2}\left(q_{2}-q_{1}\right)=0,
\end{aligned}
$$

where $q_{1}$ and $q_{2}$ are the absolute cross-wind displacements of the PS and of the TMD, respectively, $m_{s}, m_{t}, \xi_{s}, \xi_{t}$ are masses and damping coefficients and $\omega_{s}$ and $\omega_{t}$ are the undamped frequencies of the two isolated bodies. The aerodynamic force $f_{\mathrm{a}}$ is 


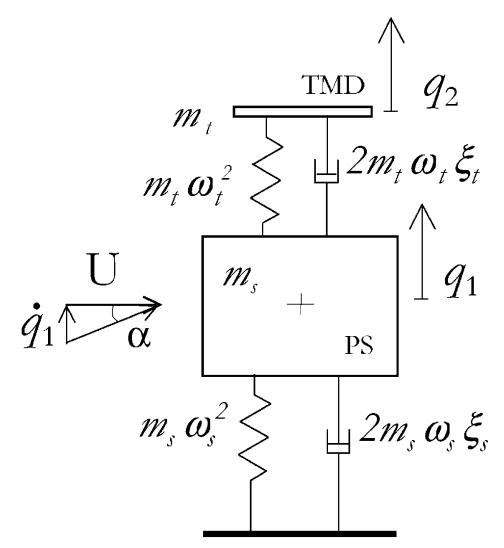

Fig. 1. Aeroelastic oscillator with tuned mass damper.

the component in the $q_{1}$-direction of the lift and drag forces; in the quasi-static regime it assumes the following expression [12]:

$$
f_{\mathrm{a}}=-\frac{1}{2} \rho U_{\mathrm{rel}}^{2} D\left(c_{\mathrm{L}} \cos \alpha+c_{\mathrm{D}} \sin \alpha\right), \quad U_{\mathrm{rel}}^{2}=\dot{q}_{1}^{2}+U^{2}, \quad \tan \alpha=\dot{q}_{1} / U,
$$

where $U$ is the uniform wind velocity, $\rho$ the air density, $D$ a typical dimension of the body, $c_{\mathrm{L}}$ and $c_{\mathrm{D}}$ are the aerodynamic coefficients, functions of the shape and of the angle of attack $\alpha$. The nonlinear force can be expressed in polynomial form by expanding Eq. (2) in Taylor series of $\dot{q}_{1} / U$, [1,2], i.e. $f_{\mathrm{a}}=\sum_{k=1}^{\infty} A_{k}\left(\dot{q}_{1} / U\right)^{k}$, where $A_{k}$ are aerodynamic force coefficients. Here, it is assumed that the cross-section of the body is symmetric with respect to the flow direction, so that even terms in the expansion vanish; moreover, powers of $\dot{q}_{1}$ higher than 3 are neglected. By introducing the following non-dimensional variables

$$
\tilde{q}_{1}=\frac{q_{1}}{D}, \quad \tilde{q}_{2}=\frac{q_{2}}{D}, \quad \gamma=\frac{\omega_{t}}{\omega_{s}}, \quad \mu=\frac{m_{t}}{m_{s}}, \quad \delta=\frac{1}{2} \frac{\rho_{\mathrm{a}} D^{2}}{m_{s}}, \quad \tilde{U}=\frac{U}{\omega_{s} D}, \quad \tilde{t}=t \omega_{s}
$$

and omitting the tilde for sake of simplicity, Eq. (1), reads

$$
\begin{aligned}
& \ddot{q}_{1}+2 \xi \dot{q}_{1}+2 \mu \gamma \xi_{t}\left(\dot{q}_{1}-\dot{q}_{2}\right)+q_{1}+\mu \gamma^{2}\left(q_{1}-q_{2}\right)=\delta \frac{A_{3}}{U} \dot{q}_{1}^{3}, \\
& \ddot{q}_{2}+2 \gamma \xi_{t}\left(\dot{q}_{2}-\dot{q}_{1}\right)+\gamma^{2}\left(q_{2}-q_{1}\right)=0,
\end{aligned}
$$

where $2 \xi=2 \xi_{s}-\delta A_{1} U$ is the apparent damping coefficient, which includes the linear part of the aerodynamic force. Eqs. (3) and (4) show that, for fixed characteristics of the PS, the system behavior depends on four parameters, the mass ratio $\mu$, the frequency ratio $\gamma$, the damping $\xi_{t}$ and the wind velocity $U$ or, equivalently, the apparent damping $\xi$. 


\section{Critical analysis}

Design methods for tuned mass damper, preventing self-excited oscillations, have been presented by various authors $[9,13,14]$. The analyses are generally devoted to describe the regions of stability of the trivial equilibrium position $\left(q_{1}, q_{2}\right)=(0,0)$ of Eq. (4) in the $\left(\xi, \xi_{t}, \gamma, \mu\right)$-parameter space, where $\xi$ is the bifurcation parameter and $\xi_{t}, \gamma, \mu$ characterize the TMD. The aim is to determine the values of the parameters that produces a desired or maximum flow-velocity $U$ at which galloping starts. In the works of Rowbottom [13] and Fujino [9] suitable sections of the stability regions are presented, focusing the attention on the first critical conditions. Some of these results are summarized and enriched in the following, where a second critical surface completing the scenario is also described.

The stability analysis of the trivial solution is performed by analyzing the spectral properties of the linearized equation,

$$
\ddot{\mathbf{q}}+\mathbf{C} \dot{\mathbf{q}}+\mathbf{K q}=\mathbf{0},
$$

where

$$
\mathbf{q}=\left\{\begin{array}{l}
q_{1} \\
q_{2}
\end{array}\right\}, \quad \mathbf{C}=\left[\begin{array}{cc}
2 \xi+2 \mu \gamma \xi_{t} & -2 \mu \gamma \xi_{t} \\
-2 \gamma \xi_{t} & 2 \gamma \xi_{t}
\end{array}\right], \quad \mathbf{K}=\left[\begin{array}{cc}
1+\mu \gamma^{2} & -\mu \gamma^{2} \\
-\gamma^{2} & \gamma^{2}
\end{array}\right] .
$$

The eigenvalues $\lambda_{i}$ of Eq. (5) are evaluated as solutions of the characteristic equation $\operatorname{det}\left[\lambda^{2} \mathbf{E}+\lambda \mathbf{C}+\mathbf{K}\right]=\mathbf{0}$ (see Appendix A); the associated right and left eigenvectors $\mathbf{u}$ and $\mathbf{v}$, necessary to the post-critical analysis, are respectively solutions of the following algebraic problems

$$
\left[\lambda^{2} \mathbf{E}+\lambda \mathbf{C}+\mathbf{K}\right] \mathbf{u}=\mathbf{0} ; \quad\left[\lambda^{2} \mathbf{E}+\lambda \mathbf{C}+\mathbf{K}\right]^{\mathrm{H}} \mathbf{v}=\mathbf{0},
$$

where $H$ denotes transpose conjugate.

For technical values of the parameters the eigenvalues are found to be complex conjugate in pairs. The stability boundaries in the parameter space are determined by requiring one or both the pairs of eigenvalues have zero real part. By imposing these conditions on the solutions of the characteristic equation some relations among the parameters is obtained. From a geometrical point of view, they describe manifolds $\mathscr{F}^{k}$ in a four-dimensional space having codimension $k(k=1,2,3)$, equal to the number of equalities imposed on the real and imaginary parts of $\lambda_{i}$. Analytical expressions of these boundary surfaces are given in Appendix A. The critical manifolds are represented in Fig. 2 for fixed value of $\mu$ in the $\left(\xi, \xi_{t}, \gamma\right)$-space. In Fig. 2a, a 3D representation of the critical manifolds, is given; in particular the existence of a peculiar point, $P^{*} \equiv\left(\xi^{*}, \xi_{t}^{*}, \gamma^{*}\right)$ at which the critical wind velocity is maximized (optimum TMD), is highlighted. In Fig. $2 \mathrm{~b}-\mathrm{d}$ three sections parallel to the reference planes, passing through the point $P^{*}$, are depicted. In particular, Fig. $2 \mathrm{~b}$ represents a section of the region at fixed $\xi=\xi^{*}$ (bold line) at which curves for different level of $\xi$ have been added. Similarly, Fig. 2c and $\mathrm{d}$ present the section at $\gamma=\gamma^{*}$ and $\xi_{t}=\xi_{t}^{*}$, with addition of some contour lines respectively. 


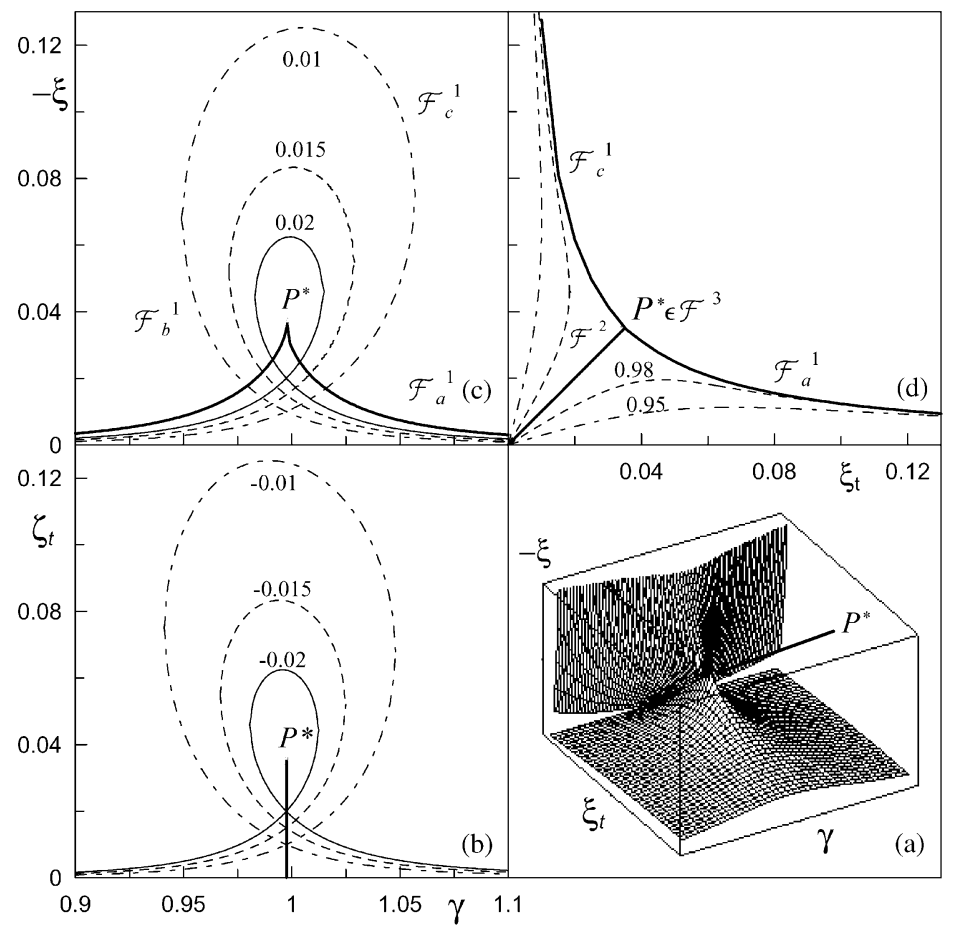

Fig. 2. Critical manifolds in the $\left(\xi, \xi_{t}, \gamma\right)$ space for $\mu \quad 0.005$ : (a) $3 \mathrm{D}$ view, (b) section at $\xi \quad \xi^{*}$, (c) section at $\gamma \quad \gamma^{*},(\mathrm{~d})$ section at $\xi_{t} \quad \xi_{t}^{*}$.

By posing $\lambda_{1,2}=2 \zeta_{1} \pm \mathrm{i} \omega_{1}$ and $\lambda_{3,4}=2 \zeta_{2} \pm \mathrm{i} \omega_{2}$, on these critical manifolds the following conditions occurs:

$$
\begin{aligned}
& \mathscr{F}_{a}^{1}: \zeta_{1}=0, \zeta_{1}^{\prime}>0, \zeta_{2}<0 ; \quad \mathscr{F}_{b}^{1}: \zeta_{1}>0, \zeta_{2}=0, \zeta_{2}^{\prime}>0 ; \\
& \mathscr{F}_{c}^{1}: \zeta_{1}=0, \zeta_{1}^{\prime}<0, \zeta_{2}>0 ; \quad \mathscr{F}^{2}: \zeta_{1}=\zeta_{2}=0, \omega_{1} \neq \omega_{2}, \zeta_{1}^{\prime}>0, \zeta_{2}^{\prime}>0 ; \\
& \mathscr{F}^{3}: \zeta_{1}=\zeta_{2}=0, \omega_{1}=\omega_{2}, \quad \zeta_{1}^{\prime}>0, \zeta_{2}^{\prime}>0 .
\end{aligned}
$$

where $\zeta_{i}^{\prime}=-\partial \zeta_{i} / \partial \xi$ are the velocities of the real part of the eigenvalues.

On the manifolds $\mathscr{F}^{1}=\left\{\mathscr{F}_{a}^{1} \cup \mathscr{F}_{b}^{1} \cup \mathscr{F}_{c}^{1}\right\}$, a forward increment of the wind velocity produce the crossing of one eigenvalue of the imaginary axis, thus leading to a simple Hopf bifurcation. In particular, on $\mathscr{F}_{a}^{1}\left(\mathscr{F}_{b}^{1}\right)$ the first (the second) eigenvalue crosses the imaginary axis with positive velocity, while on $\mathscr{F}_{c}^{1}$ a re-entry of one of the two eigenvalues occurs. On the manifolds $\mathscr{F}^{2}$ and $\mathscr{F}^{3}$ the simultaneous crossing of the imaginary axis of two pairs of eigenvalues occurs having different frequencies (non-resonant double-Hopf bifurcation) or the same frequency (1:1 resonant double Hopf bifurcation), respectively.

By summarizing, simple- and double-Hopf bifurcations are likely to occur. For high level of damping $\xi_{t}$ in the TMD or non-perfect tuning $\gamma \neq \gamma^{*}$, a simple Hopf occurs on the surface $\mathscr{F}_{a}^{1}$. Differently, for low level of damping and nearly perfect 
tuning a non-resonant double Hopf bifurcations manifests itself along the line $\mathscr{F}^{2}$. The description of the post-critical scenario around these critical conditions is the object of the following sections. The analysis of the 1:1 resonant Hopf bifurcation occurring at point $P^{*}$ is instead left for future investigation.

\section{Postcritical analysis}

The Multiple Scale Method is applied to analyze the post-critical behavior of the system around $\mathscr{F}_{a}^{1}$ and $\mathscr{F}^{2}$, according to the procedure illustrated in [16]. A generic point $P_{0} \equiv\left(\xi_{0} ; \gamma_{0} ; \xi_{t 0}\right) \in \mathscr{F}_{a}^{1}$ or $\in \mathscr{F}^{2}$ is fixed and a perturbation of one or two parameters is performed, for codimension 1 or 2 bifurcations, respectively. Namely $\xi=\xi_{0}-\varepsilon^{2} \xi_{2}, \gamma=\gamma_{0}+\varepsilon^{2} \gamma_{2}$ is posed, where $\varepsilon$ is a perturbation parameter, $\xi_{2}$ has the meaning of distinguished parameter (positive for overcritical wind velocities) and $\gamma_{2}$ of splitting parameter. Moreover, the Lagrangian coordinates are expanded in series of $\varepsilon$ as

$$
\mathbf{q}(t, \varepsilon)=\varepsilon \mathbf{q}_{1}+\varepsilon^{3} \mathbf{q}_{3}+O\left(\varepsilon^{5}\right)
$$

and several temporal scales $t_{k}=\varepsilon^{k} t(k=0,2, \ldots)$ are introduced, so that $\mathrm{d} / \mathrm{d} t=$ $\mathrm{d}_{0}+\varepsilon^{2} \mathrm{~d}_{2}+\cdots$, with $d_{k}:=\partial / \partial t_{k}$. By substituting the previous equations in the Eqs. (4) the following perturbation equations up to $\varepsilon^{3}$ are drawn:

$$
\begin{aligned}
& \left(d_{0}^{2} \mathbf{E}+d_{0} \mathbf{C}_{0}+\mathbf{K}_{0}\right) \mathbf{q}_{1}=0, \\
& \left(d_{0}^{2} \mathbf{E}+d_{0} \mathbf{C}_{0}+\mathbf{K}_{0}\right) \mathbf{q}_{3}=-d_{0} \mathbf{C}_{2} \mathbf{q}_{1}-\mathbf{K}_{2} \mathbf{q}_{1}+\mathbf{f}\left(\mathbf{q}_{1}\right)-\left(d_{1}^{2}+2 d_{0} d_{2}\right) \mathbf{q}_{1},
\end{aligned}
$$

where the matrices $\mathbf{C}_{0}$ and $\mathbf{K}_{0}$ are the damping and stiffness matrices defined by Eqs. $\left(6_{2}\right)$ and $\left(6_{3}\right)$ evaluated at the bifurcation point $P_{0}$ and the higher order-terms are

$$
\begin{gathered}
\mathbf{C}_{2}=\left[\begin{array}{cc}
-2 \xi_{2}+2 \mu \gamma_{2} \xi_{t} & -2 \mu \gamma_{2} \xi_{t} \\
-2 \gamma_{2} \xi_{t} & 2 \gamma_{2} \xi_{t}
\end{array}\right], \quad \mathbf{K}_{2}=\left[\begin{array}{cc}
2 \mu \gamma_{0} \gamma_{2} & -2 \mu \gamma_{0} \gamma_{2} \\
-2 \gamma_{0} \gamma_{2} & 2 \gamma_{0} \gamma_{2}
\end{array}\right], \\
\mathbf{f}\left(\mathbf{q}_{1}\right)=\left[\begin{array}{c}
\delta \frac{A_{3}}{U} \dot{q}_{11}^{3} \\
0
\end{array}\right] .
\end{gathered}
$$

It should be noted that, in Eq. $\left(12_{3}\right)$, the actual value $U$ of the wind velocity has been considered, instead of the bifurcation value $U_{0},[2]$. Although this procedure is inconsistent, numerical results have shown that it improves the accuracy of the solution for $U \gg U_{0}$ (see later Section 5). In the following, the simple and the nonresonant double Hopf bifurcations are analyzed.

\subsection{Simple Hopf bifurcation}

In this case $P_{0} \in \mathscr{F}_{a}^{1}$, and a single critical mode $\mathbf{u} \in \mathbb{C}$ of frequency $\omega$ exists; therefore the generating solution of Eq. (10) reads

$$
\mathbf{q}_{1}=A\left(t_{1}, t_{3}, \ldots\right) \mathbf{u} \mathrm{e}^{\mathrm{i} \omega t_{0}}+\text { c.c. }
$$


where $A$ is the complex amplitude and c.c. stands for complex conjugate terms. By substituting Eqs. (13) in (11) it holds:

$$
\left(d_{0}^{2} \mathbf{E}+d_{0} \mathbf{C}_{0}+\mathbf{K}_{0}\right) \mathbf{q}_{3}=\mathbf{b}_{1} \mathrm{e}^{\mathrm{i} \omega t_{0}}+\text { n.s.t. }+ \text { c.c. }
$$

with $\mathbf{b}_{1}$ defined in Appendix $\mathrm{B}$, depending on the $\xi_{2}$ bifurcation parameter (see Appendix B). By enforcing the solvability conditions $\mathbf{v}_{1}^{\mathrm{H}} \mathbf{b}_{1}=0$, the following ordinary differential equation in the complex amplitude $A\left(t_{1}, t_{3}, \ldots\right)$ is found:

$$
d_{2} A=C_{11} A \xi_{2}+C_{13} A^{2},
$$

where the coefficients $C_{i j}$ are defined in the Appendix B. Expressing the amplitudes in the polar form $A=\frac{1}{2} a\left(t_{2}\right) \mathrm{e}^{\mathrm{i} \varphi\left(t_{2}\right)}$ and separating the real and imaginary parts of Eqs. (15), two differential equations of the first order follow

$$
\dot{a}=-R_{11} \xi_{2} a+R_{13} a^{3}, \quad \dot{\varphi}=-I_{11} \xi_{2}+I_{13} a^{2},
$$

where $R_{i j}=\operatorname{Re}\left(C_{i j}\right)$ and $I_{i j}=\operatorname{Im}\left(C_{i j}\right)$.

Eqs. (16) admit the trivial solution $a_{0}=0$ and the non-trivial steady-state solution

$$
a_{0}^{2}=\frac{R_{11}}{R_{13}} \xi_{2}, \quad \varphi=\left[\left(-I_{11}+I_{13} \frac{R_{11}}{R_{13}}\right) \xi_{2}\right] t_{2}+\varphi_{0},
$$

respectively. Eqs. (17) describe the amplitude and the frequency correction of periodic galloping response of the system. By normalizing the critical mode as $\mathbf{u}=\left[1, \rho \mathrm{e}^{\mathrm{i} \alpha}\right]^{\mathrm{T}}$, at the leading order the motion is described by

$$
q_{1}(t)=a_{0} \cos \left(\Omega t+\varphi_{0}\right), \quad q_{2}(t)=\rho a_{0} \cos \left(\Omega t+\varphi_{0}+\alpha\right),
$$

where $\Omega=\omega+\left(I_{11}-I_{13} R_{11} / R_{13}\right) \xi_{2}$ is the nonlinear frequency.

The stability of both the trivial and non-trivial solution is governed by the variational equation

$$
\delta \dot{a}=\left(-R_{11} \xi_{2}+3 R_{13} a_{0}^{2}\right) \delta a .
$$

Since, by hypothesis, the trivial solution is stable for $\xi_{2}<0$, it is $R_{11}<0$; therefore the bifurcation described by Eq. (17) is overcritical (stable) if $R_{13}<0$ and undercritical (unstable) if $R_{13}>0$.

\subsection{Non-resonant double-Hopf bifurcation}

In this case $P_{0} \in \mathscr{F}^{2}$ and two critical modes $\mathbf{u}_{1}$ and $\mathbf{u}_{2} \in \mathbb{C}$, of frequencies $\omega_{1}$ and $\omega_{2}$, exist. Since $\omega_{1} \neq \omega_{2}$, the bifurcation is non-resonant. The generating solution of Eq. (10) is

$$
\mathbf{q}_{1}=A_{1}\left(t_{0}, t_{2}, \ldots\right) \mathbf{u}_{1} \mathrm{e}^{\mathrm{i} \omega_{1} t_{0}}+A_{2}\left(t_{0}, t_{2}, \ldots\right) \mathbf{u}_{2} \mathrm{e}^{\mathrm{i} \omega_{2} t_{0}}+\text { c.c. }
$$

With Eq. (20), Eq. (21) reads

$$
\left(d_{0}^{2} \mathbf{E}+d_{0} \mathbf{C}_{0}+\mathbf{K}_{0}\right) \mathbf{q}_{3}=\mathbf{b}_{1} \mathrm{e}^{\mathrm{i} \omega_{1} t_{0}}+\mathbf{b}_{2} \mathrm{e}^{\mathrm{i} \omega_{2} t_{0}}+\text { n.s.t. }+ \text { c.c. },
$$


where $\mathbf{b}_{1}$ and $\mathbf{b}_{2}$, defined in Appendix B, contain both the $\gamma_{2}$ and $\xi_{2}$ parameters. By enforcing the solvability conditions, namely $\mathbf{v}_{1}^{\mathrm{H}} \mathbf{b}_{1}=0$ and $\mathbf{v}_{2}^{\mathrm{H}} \mathbf{b}_{2}=0$, a set of ordinary differential equations in the complex amplitudes $A_{i}$ follows:

$$
\begin{aligned}
& d_{2} A_{1}=C_{11} A_{1} \xi_{2}+C_{12} A_{1} \gamma_{2}+C_{13} A_{1}^{2} A_{1}+C_{14} A_{1} A_{2} A_{2}, \\
& d_{2} A_{2}=C_{21} A_{2} \xi_{2}+C_{22} A_{2} \gamma_{2}+C_{23} A_{2}^{2} A_{2}+C_{24} A_{2} A_{1} A_{1}
\end{aligned}
$$

where the coefficients are defined in Appendix B. Expressing the amplitudes in polar form, $A_{1}=\frac{1}{2} a_{1}\left(t_{2}\right) \mathrm{e}^{\mathrm{i} \varphi_{1}\left(t_{2}\right)}$ and $A_{2}=\frac{1}{2} a_{2}\left(t_{2}\right) \mathrm{e}^{\mathrm{i} \varphi_{2}\left(t_{2}\right)}$ and separating the real and imaginary parts, Eqs. (22) brings to four differential equations of the first order:

$$
\begin{aligned}
& \dot{a}_{1}=\left(R_{11} \xi_{2}+R_{12} \gamma_{2}\right) a_{1}+R_{13} a_{1}^{3}+R_{14} a_{1} a_{2}^{2}, \\
& \dot{a}_{2}=\left(R_{21} \xi_{2}+R_{22} \gamma_{2}\right) a_{2}+R_{23} a_{2}^{3}+R_{24} a_{1}^{2} a_{2}, \\
& \dot{\varphi}_{1}=\left(I_{11} \xi_{2}+I_{12} \gamma_{2}\right)+I_{13} a_{1}^{2}+I_{14} a_{2}^{2}, \\
& \dot{\varphi}_{2}=\left(I_{21} \xi_{2}+I_{22} \gamma_{2}\right)+I_{23} a_{2}^{2}+I_{24} a_{1}^{2},
\end{aligned}
$$

where the coefficients $C_{i j}$ have been separated in the real and imaginary parts. Eq. (23) are uncoupled from Eq. (24) and govern the evolution of the amplitudes of the two critical modes.

The Eq. (23) admit the trivial solution $a_{1}=a_{2}=0$. However, non-trivial steadystate solutions are possible causing mono-modal or bi-modal galloping. Monomodal galloping occurs when one of the two modal amplitudes vanishes. Thus, if $a_{2}=0$, Eq. $\left(23_{2}\right)$ is identically satisfied, while Eqs. $\left(23_{2}\right)$ and $\left(24_{1}\right)$ lead to

$$
\begin{aligned}
& a_{10}^{2}=-\frac{1}{R_{13}}\left(R_{11} \xi_{2}+R_{12} \gamma_{2}\right) ; \\
& \left.\varphi_{1}=\left[\left(I_{11}-I_{13} \frac{R_{11}}{R_{13}}\right) \xi_{2}+\left(I_{12}-I_{13} \frac{R_{12}}{R_{13}}\right) \gamma_{2}\right)\right] t_{2}+\varphi_{10},
\end{aligned}
$$

respectively. Similarly, if $a_{1}=0$, Eq. $\left(23_{1}\right)$ is identically satisfied, while Eqs. $\left(23_{2}\right)$ and $\left(24_{2}\right)$ yield

$$
\begin{aligned}
& a_{20}^{2}=-\frac{1}{R_{23}}\left(R_{21} \xi_{2}+R_{22} \gamma_{2}\right) ; \\
& \left.\varphi_{2}=\left[\left(I_{21}-I_{23} \frac{R_{21}}{R_{23}}\right) \xi_{2}+\left(I_{22}-I_{23} \frac{R_{21}}{R_{13}}\right) \gamma_{2}\right)\right] t_{2}+\varphi_{10}
\end{aligned}
$$

Since $a_{1}$ and $a_{2}$ are real, Eqs. (25) and (26) hold only in half-planes of the $\left(\xi_{2}, \gamma_{2}\right)$ plane, delimited by the straight lines $s_{1}:=R_{11} \xi_{2}+R_{12} \gamma_{2}=0$ and $s_{2}:=$ $R_{21} \xi_{2}+R_{22} \gamma_{2}=0$, respectively. Finally if both $a_{1}$ and $a_{2}$ are different from zero, a bi-modal (quasi-periodic) steady-state solution exists:

$$
\begin{aligned}
& a_{1 Q}^{2}=\frac{\left(R_{14} R_{21}-R_{11} R_{23}\right) \xi_{2}+\left(R_{14} R_{22}-R_{12} R_{23}\right) \gamma_{2}}{R_{23} R_{13}-R_{24} R_{14}} \\
& a_{2 Q}^{2}=\frac{\left(R_{24} R_{11}-R_{21} R_{13}\right) \xi_{2}+\left(R_{24} R_{12}-R_{22} R_{13}\right) \gamma_{2}}{R_{23} R_{13}-R_{24} R_{14}}
\end{aligned}
$$


The domain of definition of Eqs. (27) is a sector of the $\left(\xi_{2}, \gamma_{2}\right)$-plane delimited by the straight lines $r_{1}:=\left(R_{14} R_{21}-R_{11} R_{23}\right) \xi_{2}+\left(R_{14} R_{22}-R_{12} R_{23}\right) \gamma_{2}=0$ and $r_{2}:=$ $\left(R_{24} R_{11}-R_{21} R_{13}\right) \xi_{2}+\left(R_{24} R_{12}-R_{22} R_{13}\right) \gamma_{2}=0$.

The stability of the steady-state solutions of Eqs. (23) is governed by the following variational equation:

$\left\{\begin{array}{l}\delta \dot{a}_{1} \\ \delta \dot{a}_{2}\end{array}\right\}=\left[\begin{array}{cc}\left(R_{11} \xi_{2}+R_{12} \gamma_{2}+3 R_{13} a_{10}^{2}+R_{14} a_{20}^{2}\right) & 2 R_{14} a_{10} a_{20} \\ 2 R_{24} a_{10} a_{20} & \left(R_{21} \xi_{2}+R_{22} \gamma_{2}+3 R_{23} a_{20}^{2}+R_{24} a_{10}^{2}\right)\end{array}\right]\left\{\begin{array}{l}\delta a_{1} \\ \delta a_{2}\end{array}\right\}$,

where the Jacobian $\mathbf{J}$ is evaluated at $a_{i 0}$. Some general information can be drawn for trivial and monomodal solutions, for which $\mathbf{J}$ assumes diagonal forms. In particular, for the trivial solutions $\left(a_{10}=0, a_{20}=0\right)$, is $\mathbf{J}_{\mathrm{T}}=\operatorname{diag}\left[s_{1} ; s_{2}\right]$; the trivial solution is therefore stable in a sector of the $\left(\xi_{2}, \gamma_{2}\right)$-plane delimited by the straight lines $s_{1}$ and $s_{2}$. Then, for monomodal galloping $\left(a_{10} \neq 0, a_{20}=0 ; a_{10}=0, a_{20} \neq 0\right)$, the Jacobian reads $\mathbf{J}_{1}=\operatorname{diag}\left[-2 s_{1} ;-r_{2} / R_{13}\right]$ and $\mathbf{J}_{2}=\operatorname{diag}\left[-r_{1} / R_{13} ;-2 s_{2}\right]$, respectively. Hence, one eigenvalue keeps its sign constant in the region of existence of the solution while the other eigenvalue changes sign on one of the straight lines $r_{i}$ delimiting the region of existence of the bi-modal solutions. Therefore, the stability of monomodal solution changes when the bimodal solution borns.

\section{Numerical results}

Numerical investigations have been carried out to analyze the system post-critical behavior, using both the illustrated analytical solutions and direct time-integration of the equations of motion. First, the post-critical behavior subsequent to a simple Hopf bifurcation is analyzed, in both the over- and under-critical cases. By keeping the mass ratio $\mu$ fixed, a point $P_{0} \equiv\left(\gamma, \xi_{t}, \xi\right)$ (with index 0 omitted) is chosen on the critical manifold $\mathscr{F}_{a}^{1}$ shown in Fig. 2; then the wind velocity $U$ is increased beyond its critical value. By using Eq. $\left(17_{1}\right)$, the amplitude $a_{0}$ of the limit cycle is drawn as a function of $U$. In Fig. 3a some results concerning different $\left(\gamma, \xi_{t}\right)$-pairs are illustrated for an aeroelastic oscillator with over-critical behavior of parameters $\mu=0.005$, $\xi_{s}=8.8 E-4, \delta=4.7 E-4, A_{1}=4.87, A_{3}=-421$ and compared with the path of the uncontrolled (unc-labeled curve) system. The curve $a$ is referred to an initial point $P_{0} \equiv\left(\gamma=\gamma^{*}, \xi_{t}=0.03570, \xi=-0.03495\right)$ very close to the point $P^{*} \equiv\left(\gamma^{*}=\right.$ $0.99751, \xi_{t}^{*}=0.03553, \xi^{*}=-0.03573$ ) (see Fig. 2), while curves $b$ and $c$ refer to points $P_{0} \in \mathscr{F}_{a}^{1}$ far from $P^{*}$, obtained by modifying with respect to the previous values only $\xi_{t}$ or $\gamma$, respectively. It is seen that, controlled oscillators close to the optimum values of the parameters undergo galloping at high wind velocity and experience comparatively small oscillation amplitudes. Therefore, although the optimum values have been determined to maximize only the bifurcation value, the TMD control system furnish a good performance also in the nonlinear range. Curves $b$ and $c$ illustrate the sensitivity of the nonlinear paths. It results that the system is 

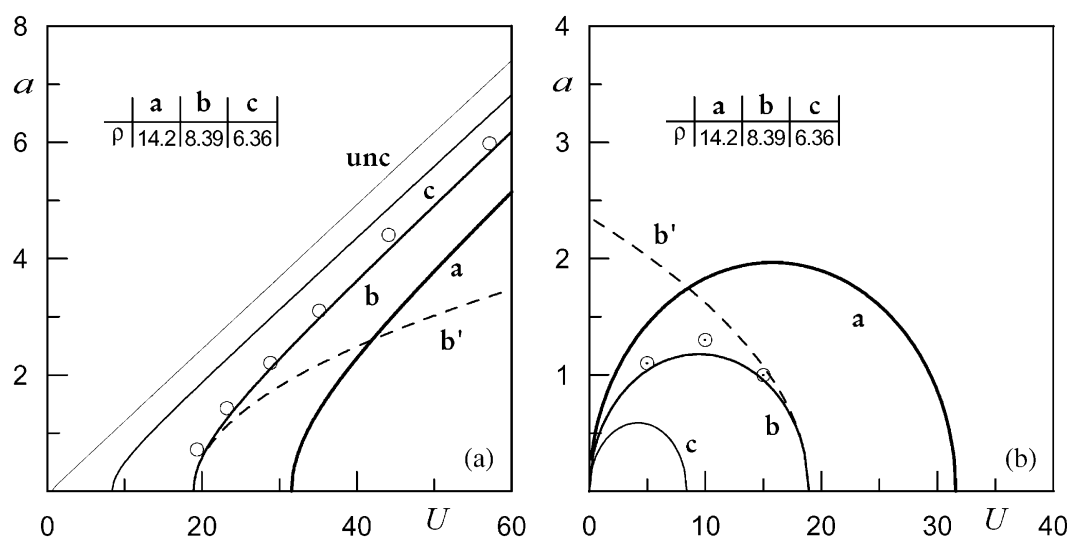

Fig. 3. PS galloping amplitude oscillations versus wind velocity subsequent to a simple Hopf bifurcation: (a) over critical behavior; (b) under critical behavior.

weakly sensitive to changes of the damping $\xi_{t}$ since, by increasing it to about $50 \%$, the critical velocity reduces to $40 \%$ and the oscillation amplitude increases to $36 \%$ at $U=40$. In contrast, the system behavior is highly sensitive to changes of the frequency ratio $\gamma$ since, by modifying it of about $\pm 5 \%$ the critical velocity reduce of $73 \%$ and the oscillation amplitude increases of $90 \%$ at $U=40$.

The analytical results have been also compared with direct numerical integration of the equation of motion (dots in Fig. 3a), and a very good agreement has been found. On the other hand, as shown by the curve $b^{\prime}$, the analytical solution entails a large error if $U_{0}$ instead of $U$ is used in Eq. $\left(12_{3}\right)$ (and consequently in the coefficient $C_{13}$ ); for this reason the inconsistent solution has been used here.

In Fig. 3 the ratio $\rho$ (see Eq. (18)) between the amplitudes of the oscillation of the TMD and the PS is also reported for the three oscillators considered. It turns out that the TMD oscillates at larger amplitudes near point $P^{*}$, i.e. it absorbs a larger amount of the total energy put in the system by the nonconservative forces, that explaining the better performance of the optimum TMD.

Fig. $3 \mathrm{~b}$ describes the system behavior in the under-critical case, obtained by changing the sign of the aerodynamic coefficient $A_{3}$, for the same three systems of Fig. 3a. Remarkable differences between the consistent solution (curve $b^{\prime}$ ) and the inconsistent one (curve b) have been found. Comparisons with direct integration of the equation of motion again show a good agreement. The amplitude of the unstable limit cycle has been numerically evaluated through backwards integration. However, an accurate treatment of the choice of the initial conditions was necessary in order to approach the unstable cycle. Indeed, numerical results have revealed the existence of a second unstable limit cycle of smaller amplitude associated with the second, noncritical, mode. Such a behavior cannot be described by a simple Hopf analysis since it is due to the effects of the second pair of eigenvalues close the critical condition.

The postcritical behavior of the system around a double Hopf bifurcation is now studied by using the analytical results of Section 4.2. In Fig. 4 the post-critical 


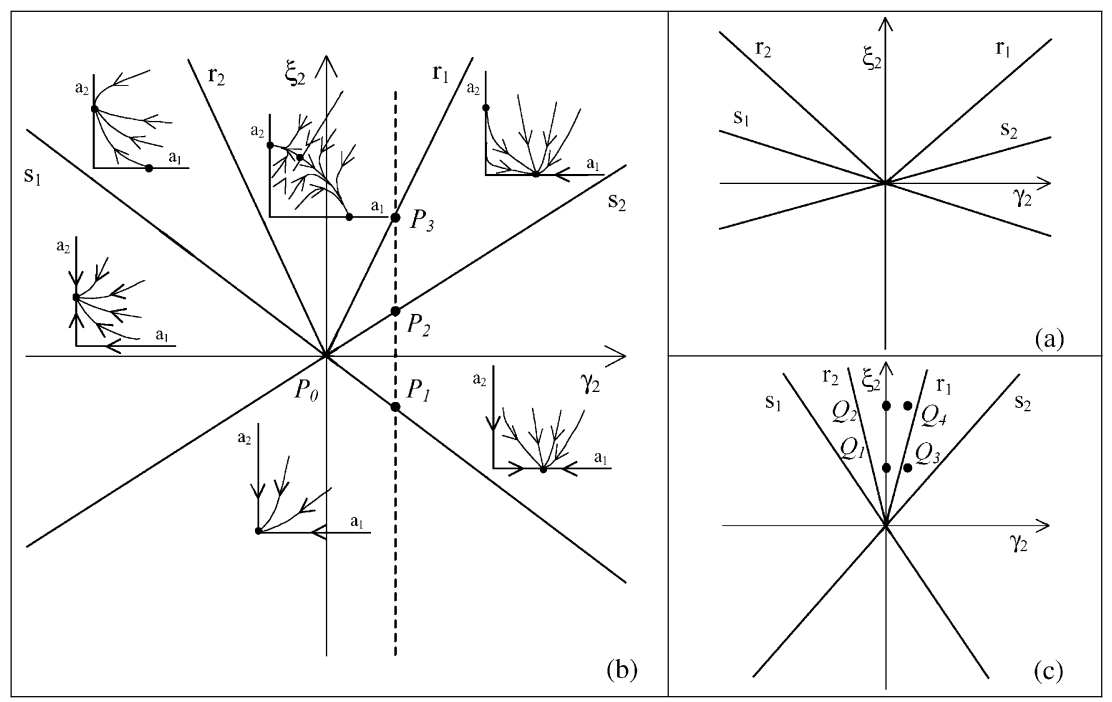

Fig. 4. Bifurcation diagram in the $\left(\xi_{2}, \gamma_{2}\right)$ plane for $\mu \quad 0.005$ : (a) $\xi_{t} \quad 0.01$; (b) $\xi_{t} \quad 0.02$; (c) $\xi_{t} \quad 0.033$.

scenario is illustrated in the $\left(\gamma_{2}, \xi_{2}\right)$-plane for three different critical points $P_{0} \in \mathscr{F}^{2}$. The two pairs of straight lines $\left(s_{1}, s_{2}\right)$, delimiting the regions of the existence of mono-modal solutions, and the lines $\left(r_{1}, r_{2}\right)$ describing the boundaries of the regions in which bi-modal solutions take place, are depicted. In each sector of the plane a sketch of the relevant phase-portrait is shown. In the lower sector the trivial solution is stable; in the two adjacent sectors, stable mono-modal, periodic, galloping occurs in one of the two modes; then, the two mono-modal solution coexist, one of them being stable and the other unstable; finally, in the upper sector, the bi-modal quasiperiodic solution appears together with the two mono-modal solution. Stability analysis shows that the quasi-periodic solution is unstable, while the periodic ones are both stable. Therefore, initial conditions decide in which of the two modes the system oscillates at regime. When the point $P_{0}$ is changed, and approaches $P^{*}$, the scenario modifies according to the sequence $a, b, c$ in Fig. 4; thus, the region of the bimodal galloping shrinks together with the region of stable trivial solution.

When the wind velocity $U$ increases from zero, a straight path parallel to the $\xi_{2}$ axis is described on the $\left(\gamma_{2}, \xi_{2}\right)$-plane. A sample path has been chosen in Fig. $4 \mathrm{~b}$, crossing the lines $s_{1}, s_{2}$ and $r_{1}$ at the points $P_{i}(i=1, \ldots, 3)$. The relevant amplitudes of the limit cycles vs. the wind velocity, as evaluated by Eqs. (25)-(27), are drawn in Fig. 5a. For increasing $U$, a stable monomodal galloping of amplitude $a_{10}$ starts at $P_{1}$; then, an unstable mono-modal galloping of amplitude $a_{20}$ is triggered at $P_{2}$; this gains stability at $P_{3}$, where an unstable quasi-periodic solutions $\left(a_{1 Q}, a_{2 Q}\right)$ bifurcates from it. By comparing the stable $a_{10}$ and $a_{20}$ curves with the unc-labeled curve of Fig. 3a, relevant to the uncontrolled case, it is concluded that even in the cases in which the TMD entails the occurence of a double-Hopf bifurcations, it reduces the amplitudes of the limit cycle. The TMD has therefore a beneficial effect. 


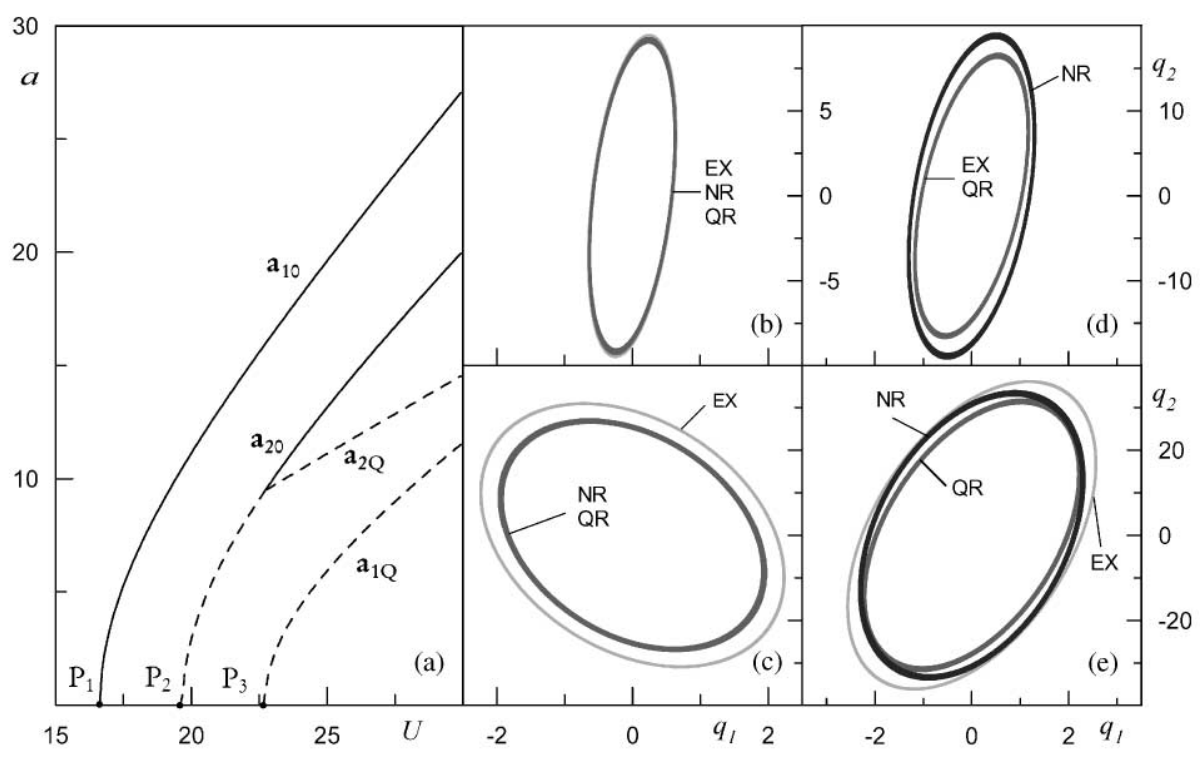

Fig. 5. PS galloping oscillations subsequent to a double Hopf bifurcation: (a) amplitudes of limit cycle vs. wind velocity (NR solution); (b) (d) projection of the limit cycles on the configuration plane (NR and QR analytical solutions; EX direct integration).

In order to evaluate the accuracy of the analytical predictions, some stable limit cycles described by the first approximation, Eq. (20), have been compared with direct integrations of motion. Fig. 5b-e shows the trajectories at the regime on the configuration $\left(q_{1}, q_{2}\right)$-plane, orderly for the four $Q_{i}$ points marked in Fig. $4 \mathrm{c}$. In each figure, NR curves (non-resonant solution) refer to analytical results, EX curves to exact numerical solution, while QR (quasi-resonant solution) will be commented later. At the $Q_{1}$-point a very good approximation is found; however, it gets worse when the wind velocity is increased $\left(Q_{2}\right.$-point), due to the fact that higher-order harmonics, present in the numerical solution and absent in the analytical solution, become significant. In this two cases, due to the choice of the initial conditions, the system oscillates in two different modes. A slightly less good accordance is found at $Q_{3}$ and $Q_{4}$, i.e. for non-perfect tuning $\gamma \neq \gamma^{*}$; the approximation is however satisfactory.

\section{Concluding remarks}

The effects of a tuned mass damper on the behavior of a SDOF bluff body exposed to a steady flow and undergoing galloping oscillations, have been analyzed both on the critical and post-critical range. The study of the critical boundaries in the parameter space has evidenced the existence of different manifolds on which single 
and double-Hopf bifurcations occur. The post-critical behavior of the system, subsequent to a simple-Hopf and a non-resonant double-Hopf have been analyzed through a perturbation method. It has been concluded that the TMD has a benificial effect also on the post-critical behavior, since it generally reduces the amplitude of the galloping oscillations. The conclusions obtained here, however, should be corroborated by a wider analysis, able to clarify some points emerged during the present study, according to the following discussion.

Numerical results obtained by direct integration of the equations of motion have shown that, even in the simple-Hopf bifurcation, the passive pair of the eigenvalues may play an important role in the description of the system behavior, as evident in the under-critical case. A deeper analysis should take into account the effects of the (small) distance of the passive eigenvalues from the immaginary axis. Similar problems exist in the analysis of the double-Hopf bifurcation occurring on $\mathscr{F}^{2}$. In Section 4.2 it was analyzed by considering distinct critical eigenvalues (non-resonant solution, NR). Indeed, due to the closeness of the eigenvalues (that increasingly approach each other until they coalesce at point $P^{*} \equiv \mathscr{F}^{3}$ ), it is expected that some interaction between the frequencies may occur even on $\mathscr{F}^{2}$. It would be desiderable, to evaluate the extension of the region of resonance around $\mathscr{F}^{3}$; however, such analysis is out of the scope of this paper and has been left for future investigation. At present the problem has been tentatively attacked along the lines illustrated in [17]. A so-called quasi-resonant solution $(\mathrm{QR})$ has been built up taking the same generating solution, Eq. (20) of the NR case, but assuming now $\omega_{2}=\omega_{1}+\varepsilon \sigma$, where $\sigma$ is a third parameter, in addition to $\xi_{2}$ and $\gamma_{2}$, describing the codimension-3 bifurcation. The QR solution is based on the hypothesis that the eigenvectors $\mathbf{u}_{1}$ and $\mathbf{u}_{2}$ can be assumed distinct when their frequencies $\omega_{1}$ and $\omega_{2}$ are still very close each other; however, it breaks down when $P_{0} \equiv P^{*}$, where $\mathbf{u}_{1} \equiv \mathbf{u}_{2}$ and the Jacobian becomes defective (nillpotent). The procedure leads to three coupled differential equations similar to that of [17], governing the evolution of $a_{1}, a_{2}$ and a phase combination. The most remarkable difference with the NR solution is that, in some region of the parameter space, the $Q R$ solution admits a bi-modal stable periodic motion. The QR solution has been used for comparison with the NR-solution and the EXsolution in Fig. 5b-e. In Fig. 5b and $c$ it is seen that the NR and QR are almost coincident, while in Fig. 5d and e the QR furnishes a better approximation. This behavior is explained by the emergence of the stable bi-modal solution. It permits to capture the modification of the critical mode, displayed by the rotation of the ellipses in Fig. $5 \mathrm{~d}$ and e that cannot be described by the NR solution. It is concluded that a more refined analysis would require to study the neighbourhood of $P^{*}$ as a perturbation of a 1:1 resonant (defective) Hopf bifurcation. Work is in progress on this matter.

\section{Acknowledgements}

This work was partially supported by Ministero della Universitá e Ricerca Scientifica e Tecnologica under the project COFIN99. 


\section{Appendix A}

The characteristic equation of the differential equation (5) has the following expression:

$$
\lambda^{4}+2\left(\xi+\gamma \xi_{t}+\mu \gamma \xi_{t}\right) \lambda^{3}+\left(\gamma^{2}+1+4 \gamma \xi \xi_{t}+\mu \gamma^{2}\right) \lambda^{2}+2 \gamma\left(\xi_{t}+\gamma \xi\right) \lambda+\gamma^{2}=0 .
$$

By posing $\lambda_{1,2}=2 \zeta_{1} \pm \mathrm{i} \omega_{1}$ and $\lambda_{3,4}=2 \zeta_{2} \pm \mathrm{i} \omega_{2}$ and requiring $\zeta_{1}=0$, an $\mathscr{F}^{1}$ codimension-1 manifold is found. By requiring $\zeta_{1}=\zeta_{2}=0$ alone or together with $\omega_{1}=\omega_{2}$, an $\mathscr{F}^{2}$ codimension-2 and an $\mathscr{F}^{3}$ codimension-3 manifolds follow, respectively. They are defined as

$$
\begin{gathered}
\mathscr{F}^{1} \equiv\left\{\begin{array}{l}
\gamma \xi^{4}+\left[1+(7+3 \mu) \gamma^{2}\right] \xi_{t} \xi^{3}+\left[\mu \gamma^{3}+\left(7+10 \mu+3 \mu^{2}\right) \gamma^{3} \xi_{t}^{2}+\xi_{t}^{2}(7+3 \mu) \gamma\right] \xi^{2} \\
+\left[1-2 \gamma^{2}+\left(7+10 \mu+3 \mu^{2}\right) \gamma^{2} \xi_{t}^{2}+\left(1+3 \mu+3 \mu^{2}+\mu^{3}\right) \gamma^{4} \xi_{t}^{2}+\left(\mu^{2}+2 \mu+1\right) \gamma^{4}\right] \xi_{t} \xi \\
+\left(1+3 \mu+3 \mu^{2}+\mu^{3}\right) \gamma^{3} \xi_{t}^{4}+\mu \gamma \xi_{t}^{2}=0
\end{array}\right. \\
\mathscr{F}^{2} \equiv\left\{\begin{array} { l } 
{ \xi = - \xi _ { t } \sqrt { } 1 + \mu , } \\
{ \gamma = 1 / \sqrt { } 1 + \mu , }
\end{array} \mathscr { F } ^ { 3 } \equiv \left\{\begin{array}{l}
\xi=-\frac{1}{2} \sqrt{ } 2-2 \sqrt{ } 1 /(1+\mu) \sqrt{ } 1+\mu, \\
\xi_{t}=\frac{1}{2} \sqrt{ } 2-2 \sqrt{ } 1 /(1+\mu), \\
\gamma=\sqrt{ } 1 /(1+\mu) .
\end{array}\right.\right.
\end{gathered}
$$

\section{Appendix B}

The vectors appearing in Eqs. (21) are

$$
\begin{aligned}
& \mathbf{b}_{1}=-\mathrm{i} \omega_{1} A_{1} \mathbf{C}_{2} \mathbf{u}_{1}-A_{1} \mathbf{K}_{2} \mathbf{u}_{1}+3 \mathrm{i} \omega_{1}^{3} A_{1}^{2} A_{1} \mathbf{f}_{1}+6 \mathrm{i} \omega_{1} \omega_{2}^{2} A_{1} A_{2} A_{2} \mathbf{f}_{2}-2 \mathrm{i} \omega_{1} \mathbf{u}_{1} d_{2} A_{1}, \\
& \mathbf{b}_{2}=-\mathrm{i} \omega_{2} A_{2} \mathbf{C}_{2} \mathbf{u}_{2}-A_{2} \mathbf{K}_{2} \mathbf{u}_{2}+3 \mathrm{i} \omega_{2}^{3} A_{2}^{2} A_{2} \mathbf{f}_{3}+6 \mathrm{i} \omega_{2} \omega_{1}^{2} A_{2} A_{1} A_{1} \mathbf{f}_{4}-2 \mathrm{i} \omega_{2} \mathbf{u}_{2} d_{2} A_{2},
\end{aligned}
$$

where

$$
\begin{aligned}
& \mathbf{f}_{1}=\left\{\begin{array}{c}
\frac{\delta A_{3}}{U} u_{11}^{2} u_{11} \\
0
\end{array}\right\}, \quad \mathbf{f}_{2}=\left\{\begin{array}{c}
\frac{\delta A_{3}}{U} u_{11} u_{21} u_{21} \\
0
\end{array}\right\}, \\
& \mathbf{f}_{3}=\left\{\begin{array}{c}
\frac{\delta A_{3}}{U} u_{21}^{2} u_{21} \\
0
\end{array}\right\}, \quad \mathbf{f}_{4}=\left\{\begin{array}{c}
\frac{\delta A_{3}}{U} u_{21} u_{11} u_{11} \\
0
\end{array}\right\} .
\end{aligned}
$$

The vector $\mathbf{b}_{1}$ in Eq. (14) is given by Eq. (31) in which $A_{2}=0$ is posed. The expressions of the coefficients in Eqs. (15) and (22) are

$$
C_{11}=v_{11} u_{11}, \quad C_{12}=\left(\xi_{t}-\mathrm{i} \gamma_{0} / \omega_{1}\right)\left[v_{12}\left(u_{11}-u_{12}\right)+\mu v_{11}\left(u_{12}-u_{11}\right)\right],
$$




$$
\begin{aligned}
& C_{13}=\frac{3}{8} \frac{\delta A_{3}}{U} \omega_{1}^{2} \bar{v}_{11} u_{11}^{2} \bar{u}_{11}, \quad C_{14}=\frac{3}{4} \frac{\delta A_{3}}{U} \omega_{2}^{2} \bar{v}_{11} u_{11} u_{21} \bar{u}_{21}, \\
& C_{21}=\bar{v}_{21} u_{21}, \quad C_{22}=\left(\xi_{t}-\mathrm{i} \gamma_{0} / \omega_{2}\right)\left[\bar{v}_{22}\left(u_{21}-u_{22}\right)+\mu \bar{v}_{21}\left(u_{22}-u_{21}\right)\right], \\
& C_{23}=\frac{3}{8} \frac{\delta A_{3}}{U} \omega_{2}^{2} \bar{v}_{21} u_{21}^{2} \bar{u}_{21}, \quad C_{24}=\frac{3}{4} \frac{\delta A_{3}}{U} \omega_{1}^{2} \bar{v}_{21} u_{21} u_{11} \bar{u}_{11} .
\end{aligned}
$$

In Eqs. (32) and (33), $\mathbf{u}_{k}=\left\{u_{k 1}, u_{k 2}\right\}^{\mathrm{T}}$ and $\mathbf{v}_{k}=\left\{v_{k 1}, v_{k 2}\right\}^{\mathrm{T}}(k=1,2)$ are the right and the left eigenvectors defined by Eq. (7), an overbar denotes the complex conjugate and ()$^{\mathrm{H}}$ the transpose conjugate.

\section{References}

[1] G.V. Parkinson, N.P.H. Brooks, On the aeroelastic instability of bluff cylinders, Journal of Applied Mechanics 28 (1961) 252-258.

[2] M. Novak, Aeroelastic galloping of prismatic bodies, Engineering of Mechanics Division, ASCE 96 (1969) 115-142.

[3] R.M. Corless, G.V. Parkinson, A model of the combined effects of vortex-induced oscillation and galloping, Journal of Fluids and Structures 2 (1988) 203-220.

[4] T.T. Soong, G.F. Dargush, Passive Energy Dissipation Systems in Structural Engineering, Wiley, New York, 1997.

[5] A. Larsen, Vortex-induced response of bridges and control by tuned mass dampers, in: Moan et al. (Eds.), Structural Dynamics - EURODYN'93, A.A. Balkema, Rotterdam, 1993, 1003-1010.

[6] A. Larsen, E. Svensson, H. Andersen, Design aspects of tuned mass dampers for the Great Belt East Bridge approach span, Journal of Wind Engineering and Industrial Aerodynamics 54-55 (1993) 413-426.

[7] V. Gattulli, R. Ghanem, Adaptive control of flow-induced oscillations including vortex effects, International Journal of Non-Linear Mechanics 34 (5) (1999) 853-868.

[8] G.B. Warburton, Optimal absorber parameters for various combinations of response and excitation parameters, Earthquake Engineering and Structural Dynamics 10 (1982) 381-401.

[9] Y. Fujino, M. Abé, Design formulas for tuned mass dampers based on a perturbation technique, Earthquake Engineering and Structural Dynamics 22 (1993) 833-854.

[10] R. Rana, T.T. Soong, Parametric study and simplified design of tuned mass dampers, Engineering Structures 20 (3) (1998) 193-204.

[11] Y. Fujino, P. Warnitchai, M. Ito, Suppression of galloping of bridge tower using tuned mass damper, Journal of the Faculty of Engineering, The University of Tokyo 38 (2) (1985) 49-73.

[12] R.D. Blevins, Flow-Induced Vibration, 2nd Edition, Van Nostrand Reinhold, New York, 1990.

[13] M.D. Rowbottom, The optimization of mechanical dampers to control self-excited galloping oscillations, Journal of Sound and Vibration 75 (4) (1981) 559-576.

[14] M. Abdel-Rohman, Design of tuned mass dampers for suppression of galloping in tall prismatic structures, Journal of Sound and Vibration 171 (3) (1994) 289-299.

[16] A. Luongo, A. Paolone, Perturbation methods for bifurcation analysis from multiple nonresonant complex eigenvalues, Nonlinear Dynamics 14 (1997) 193-210.

[17] A. Luongo, A. Paolone, G. Piccardo, Postcritical behavior of cables undergoing two simultaneous galloping modes, Meccanica 33 (1998) 229-242. 